\title{
Hypertrophic lichen planus on the face
}

\section{Mouna Korbi', Jacem Rouatbi', Manel Njima², Hichem Belhadjali', Monia Youssef', Jameleddine Zili ${ }^{1}$}

${ }^{1}$ Dermatology Department, Fattouma Bourguiba University Hospital, University of Medicine, Univesity of Monastir, Tunisia, ${ }^{2}$ Anatomopathology Department, Fattouma Bourguiba University Hospital, University of Medicine, Univesity of Monastir, Tunisia

Corresponding author: Dr. Mouna Korbi, E-mail: korbimouna68@gmail.com

Sir,

A 45-year-old man presented with a six-year-history of multiple itchy lesions on his face. The man was otherwise in a good health. Dermatological examination revealed purplish hyperkeratotic plaques with atrophic center, on the face involving the external auditory canal of the left ear (Fig. 1). We noted different age lesions (Fig. 2). There was no mucosal involvement. The rest of examination was normal. The histological examination of skin biopsy revealed a thin acanthosic epidermis with orthohyperkeratosis wedge-shaped hypergranulosis with few necrotic keratinocytes. Dermis has a lichenoid inflammatory infiltrate. We noted lymphocytes exocytosis in the basal layers of the epidermis. The dermis has elastosis lesions (Fig. 3). Direct immunofluorescence study was negative. The diagnosis of hypertrophic lichen planus on the face, involving the external auditory canal, was established. The patient was treated by highpotency corticosteroids resulting in a rapid clinical improvement.

Hypertrophic lichen planus (HLP) is a rare subtype of lichen planus (LP) that typically affects middleaged adults of both genders, mostly those with darker skinned [1-3]. It is characterized by hyperkeratotic itchy papules and plaques, involving commonly the extremities, especially the anterior legs, also the upper extremities and trunk $[2,3]$. Welsh et al described « igneous rock sign » as characteristic clinical sign of HLP as its resemblance to the extrusive forms of igneous rock [3]. In our case, the patient had HLP on his face including the external auditory canal. This location, to the best of our knowledge, has never

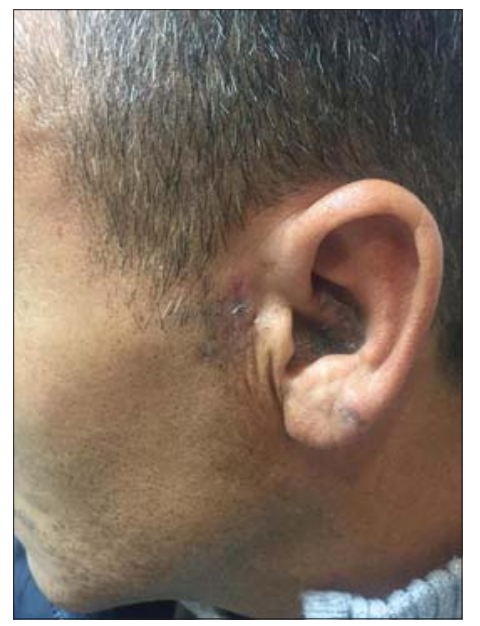

Figure 1: Purplish hyperkeratotic plaques on the external auditory canal of the left ear.

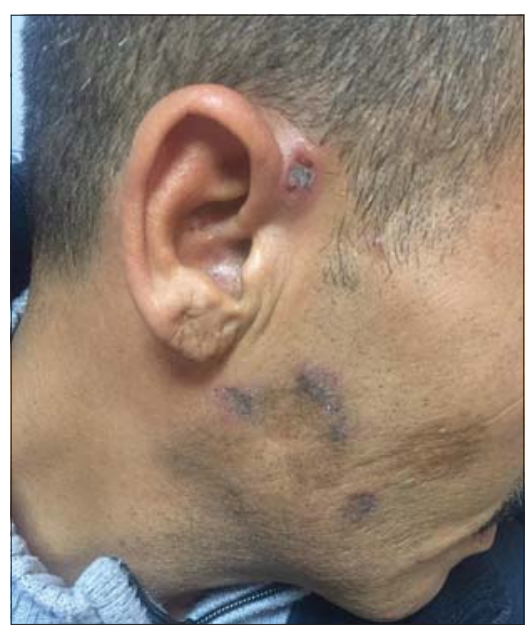

Figure 2: Multiple different age lesions with hyperkeratotic purplish plaque on the face.

been described before. LP of the external auditory is a rare clinical presentation with only around twenty

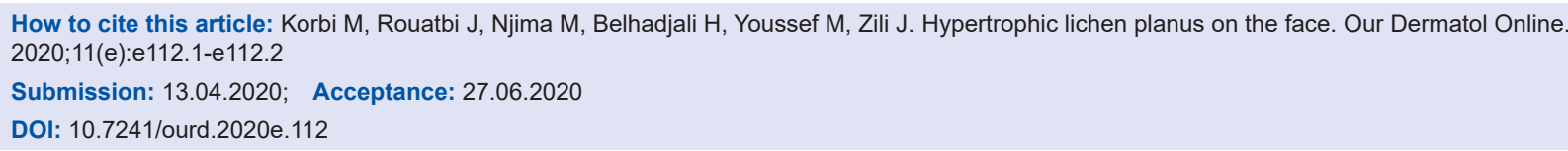




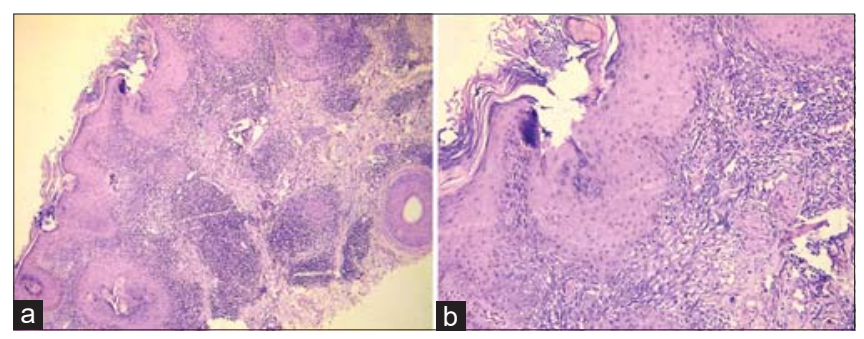

Figure 3: (a) A thin acanthosic epidermis with orthohyperkeratosis wedge-shaped hypergranulosis with inflammatory infiltrate in dermis (Hematoxylin eosin $\times 100$ ); (b) A lichenoid inflammatory infiltrate, in dermis, with lymphocytes exocytosis in the basal layers of the epidermis and elastosis lesions (Hematoxylin eosin $\times 400$ )

cases $[4,5]$. Moreover, HLP of the external auditory canal has been described in only one case without face involvement [4]. The clinical presentation of our patient can simulate other diseases such as verrucous lupus erythematosus [6]. So, a careful examination of the skin including nails, oral cavity and scalp should be performed in order to identify other suggestive clinical lesions of LP. The histopathological examination is the cornerstone of diagnosis showing typical aspects of HLP. In our case, we note the presence of a slight degree of solar elastosis that may suggest the sunlight as a precipitating factor of HLP. Treatment options for HLP are similar to those of classic LP [7]. High-potency corticosteroids are considered a firstline therapy with excellent results as the case of our patient. Phototherapy and topical or systemic immunomodulators, such as cyclosporine-analogues and retinoid [7].

\section{CONSENT}

The examination of the patient was conducted according to the Declaration of Helsinki principles.

The authors certify that they have obtained all appropriate patient consent forms. In the form the patient(s) has/have given his/her/ their consent for his/her/their images and other clinical information to be reported in the journal. The patients understand that their names and initials will not be published and due efforts will be made to conceal their identity, but anonymity cannot be guaranteed.

\section{REFERENCES}

1. Gorouhi F, Davari P, Fazel N. Cutaneous and mucosal lichen planus: a comprehensive review of clinical subtypes, risk factors, diagnosis, and prognosis. Scien World J. 2014;2014:742826.

2. Weston G, Payette M. Update on lichen planus and its clinical variants. Int J Womens Dermatol. 2015;1:140-9.

3. Welsh JP, Skvarka CB, Allen HB. A novel visual clue for the diagnosis of hypertrophic Lichen Planus. Arch Dermatol. 2006;142:954.

4. Kosec A, Kostic M, Ajduk J, Ries M. Hypertrophic recurring lichen planus of the the external auditory canal. Eur Ann Otorhinolaryngol Head Neck Dis. 2019;136:123-6.

5. Sartori-Valinotti JC, Bruce AJ, Krotova Khan Y, Beatty CW. 10-Year review of otic lichen planus: the Mayo Clinic Experience. JAMA Dermatol. 2013;149:1082-6.

6. Riahi RR, Cohen PR. Hypertrophic lichen planus mimicking verrucous lupus erythematosus. Cureus. 2018;10:e3555.

7. Alamri A, Alsenaid A, Ruzicka T, Wolf R. Hypertrophic lichen planussuccessful treatment with acitretin. Dermatol Ther. 2016;29:173-6.

Copyright by Mouna Korbi, et al. This is an open access article distributed under the terms of the Creative Commons Attribution License, which permits unrestricted use, distribution, and reproduction in any medium, provided the original author and source are credited.

Source of Support: Nil, Conflict of Interest: None declared. 